\title{
Association of Serum Triglycerides in Patients with Ischaemic Stroke Admitted in Hospital with type-2 Diabetes Patients
}

\author{
NIRMALENDU BIKASH BHOWMIK ${ }^{1}$, DILRUBAALAM ${ }^{2}$, MD RASHEDUL ISLAM ${ }^{3}$, RUMANA HABIB ${ }^{4}$, \\ AMINUR RAHMAN ${ }^{5}$, ZAHID HASSAN $^{6}$, MD.AMIRUL HAQUE $^{7}$
}

\begin{abstract}
:
Background and Aims: Diabetes mellitus and dyslipidemia, in particular triglyceridemia pose independent risk factors of stroke. Hypertriglyceridemia implicated in the pathogenesis of ischemic stroke by imparting endothelial dysfunction, oxidative stress and lowering fibrinolytic activity. This study was aimed to explore risk incurred by blood triglyceride level for ischemic stroke in type 2 diabetic patients. Materials and Methods: A total number of 80 [50 with acute ischemic stroke and 30 without stroke] type 2 diabetic patients consecutively admitted in the neurology department, during the period of April to September 2012, fulfilling the recruitment criteria were included in the study. Ischemic stroke was confirmed by CT-scan. Informed written consent from the legal attendant of each patient was obtained. Data regarding clinicobiochemical and images studies were retrieved from patient's record form. Results: Male to female ratio was 1.2:1 of the study subjects. Mean $( \pm S D)$ age (yrs) was $61.0 \pm 10.6$ in patients with ischemic stroke (Group I) and $57.0 \pm 12.3$ in patients without stroke (Group II). Risk factors like BMI, lifestyle, smoking, alcohol intake did not show any statistical significance with incidence of ischemic stroke. Mean ( $\pm S D)$ triglyceride (mg/ dl) was $241 \pm 56$ and $217 \pm 102$ in Group I and Group II respectively ( $p=0.024)$. Eighty eight percent patients had triglyceride $150 \mathrm{mg} / \mathrm{dl}$ in Group I and 70 percent in Group II. Triglyceride level (mean $\pm S D, \mathrm{mg} / \mathrm{dl})$ was significantly higher $(335 \pm 101)$ in overweightobese patients (BMI $25 \mathrm{Kg} / \mathrm{m}^{2}$ ) compared to those (232 \pm 68 ) with normal body weight $\left(B M l<25 \mathrm{Kg} / \mathrm{m}^{2}\right)$. Triglyceride level did not show statistical difference among patients having habit of smoking or not. Mean $( \pm S D)$ cholesterol $(\mathrm{mg} / \mathrm{dl}$, ( $\pm S D)$ was $197 \pm 62$ and $165 \pm 26$ in Group I and Group II respectively ( $p=0.009)$. Mean $( \pm S D) L D L-c(m g / d l)$ was $101 \pm 45$ and $98 \pm 42$ in Group I (43.8 \pm 34.4$)$ compared to Group II $(60.2 \pm 15.6)(p=0.017)$. Patients with atherosclerotic changes had significantly higher triglyceride (mean $\pm S D$, $\mathrm{mg} / \mathrm{dl})$ level (338 \pm 155$)$ compared to those without $(228 \pm 89)(p=0.047)$. Conclusions: Data concluded that hypertriglyceridemia is relatively common among the diabetic patients even in patients with apparently good glycemic control and possibly incur added risk for ischemic stroke in these patients. However, further studies are needed involving optimum number of patients to substantiate this finding and conclusively comment on the issue and to design effective prevention program to reduce the cerebrovascular morbidity and mortality.
\end{abstract}

Key words: Ischemic stroke, hypertriglyceridemia, type 2 diabetes mellitus.

Introduction:

Stroke is the third most common cause of death in the developed world after cancer and ischemic heart disease ${ }^{1}$. It may be due to either ischemia or haemorrhage. Among the patients presenting with stroke $85 \%$ have sustained cerebral infarction due to inadequate blood flow to part of the brain and remainder are caused by intracerebral hemorrhage. Cerebral infarction is mostly due to thromboembolic disease secondary to atherosclerosis in the major

1. Associate Professor of Neurology, BIRDEM General Hospital \& Ibrahim Medical College, Shahbag, Dhaka-1000.

2. Medical Officer, Department of Neurology, BIRDEM General Hospital \& Ibrahim Medical College, Shahbag, Dhaka-1000.

3. Registrar, Department of Neurology, Neurology, BIRDEM General Hospital \& Ibrahim Medical College, Shahbag, Dhaka- 1000.

4. Registrar, Department of Neurology, Neurology, BIRDEM General Hospital \& Ibrahim Medical College, Shahbag, Dhaka- 1000.

5. Associate Professor, Department of Neurology, BIRDEM General Hospital \& Ibrahim Medical College, Shahbag, Dhaka-1000.

6. Professor, Dept of Physiology \& Molecular Biology, Bangladesh University of Health Sciences (BUHS), Mirpur-1, Dhaka-1216

7. Professor of Neurology, BIRDEM General Hospital \& Ibrahim Medical College, Shahbag, Dhaka-1000. 
extracranial arteries (carotid artery and aortic arch). About $20 \%$ of infarctions are due to embolism from heart and a further $20 \%$ are due to intrinsic disease of small perforating vessels producing lacunar infarctions. The risk factors for ischemic stroke reflect the risk factor for underlying vascular disease. Perhaps $5 \%$ are due to rare causes, including vasculitis, endocarditis and cerebral venous diseases ${ }^{1}$.

Number of independent risk factors for ischemic stroke has been implicated. The most common factors include hypertension, diabetes mellitus, smoking, atrial fibrillation, coronary artery disease, and disorders of lipid metabolism. Epidemiologic studies suggest that elevated total cholesterol and low-density lipoprotein cholesterol (LDL-c), as well as low levels of high-density lipoprotein cholesterol (HDL-c) are possible risk factors for ischemic stroke 2,3 . However, consensus regarding the significance of hypertriglyceridemia as an independent risk factor for ischemic stroke still lacking.

Hypertriglyceridemia found to be one of the features of dyslipidemia seen in type 2 diabetes mellitus. Patients with poorly controlled diabetes have higher triglyceride levels than those who have the condition well controlled. Postprandial hyperlipidemia in diabetics appears to be prolonged, which means that the arteries are exposed to atherogenic particles for extended periods of time $e^{4,5}$. Postprandial hypertriglyceridemia in diabetic patients was found to produce endothelial dysfunction, oxidative stress due to lipid-derived free radicals, and impairment of endothelium-dependent vasodilatation ${ }^{6}$. Triglyceriderich lipoproteins, including very-low-density lipoprotein and intermediate-density lipoprotein, in addition to LDL-c particles, become trapped in blood vessel walls and have been demonstrated in human atherosclerotic plaques ${ }^{7}$. Chronic hypertriglyceridemia was independently associated with endothelial dysfunction in an observational study of patients with normal LDL-c ${ }^{8}$. Increased expression of adhesion cell molecules is considered to be a marker of endothelial cell dysfunction ${ }^{9}$. An increase in cell adhesion molecules has been noted in patients with hypertriglyceridemia ${ }^{9,10}$.

Another potential mechanism by which hypertriglyceridemia may contribute to atherosclerosis is through its association with elevated C-reactive protein (CRP). Elevated CRP levels have been found to be associated with elevated triglyceride levels ${ }^{11}$. In humans, carotid intimamedia thickness measures are considered reliable markers for early atherosclerosis. Increased carotid intima-media thickness has been found to be associated with an elevation of inflammatory markers, fibrinogen levels and circulating adhesion molecules, each of which has been associated with hypertriglyceridemia ${ }^{11}$. More recently Teno et al(2000) have shown the association of postprandial hypertriglyceridemia with carotid intima-media thickness in a cohort of 61 patients with type 2 diabetes. The investigators found that those with the highest postprandial triglyceride levels had the greatest degree of carotid intima-media thickness, as measured by ultrasound ${ }^{12}$.

Hypertriglyceridemia may also contribute to cerebrovascular disease through its effects on thrombosis. This effect is produced by thrombogenic alterations of the coagulation system as well as elevations in plasma viscosity. Hyperviscosity due to hypertriglyceridemia may contribute to endothelial dysfunction, tissue ischemia and chylomicronemia ${ }^{13}$. This effect is greater than that for LDL-c, supporting the greater contribution of triglycerides to plasma viscosity ${ }^{14}$. Elevated levels of triglycerides (as well as fibrinogen, total protein, LDL-c and total cholesterol) correlated positively and independently with elevated plasma viscosity ${ }^{13}$ and hypertriglyceridemia thus increase the risk of ischemic stroke by inducing a prothrombotic state through its effects on coagulation and plasma viscosity. The Copenhagen City Heart prospective Study on 19,698 men and women demonstrated strong linear association between nonfasting triglyceride levels and cerebral ischemic events like ischemic sclerosis and transient ischemic $\operatorname{attack}^{15,16}$.

The relation between high triglyceride level and ischemic stroke is not clear as it is in coronary heart disease. The role of high concentration of serum triglyceride as a risk factor for stroke is still undecided at the present time. Patient with uncontrolled diabetes usually have high triglyceride level and diabetes itself is a risk factor for stroke. 
There are limited studies on risk factor analysis of stroke in Bangladesh. Present study is designed to evaluate, is there any relationship of hypertriglyceridemia with ischemic stroke in diabetic patient. Knowledge obtained from this study may help in recognizing the magnitude of the disease problem, and to take necessary measures to treat ischemic stroke patient with diabetes and hypertrigiyceridemia in the community. More over it will provide base line information for further research for further study.

\section{Materials and Methods:}

This observational study was carried out in the department of Neurology, BIRDEM General Hospital during the period of April-September 2012. Patients with acute ischemic stroke with type 2 diabetes mellitus consecutively admitted in the department of neurology, BIRDEM General Hospital, and fulfilling the recruitment criteria were included in the study. A representative number of type 2 diabetic patients admitted in the hospital without stroke served as controls.

\section{Recruitment criteria}

Inclusion criteria

Type 2 diabetic patients, age range 20-80 yrs admitted in the hospital with clinical feature of acute ischemic stroke, confirmed by imaging study (CT/ MRI scan), their legitimate attendant(s) consented for entry in the study being detailed brief about its purpose and nature, were recruited.

\section{Exclusion criteria}

Type 2 diabetic patients with signs of previous or recent hemorrhagic stroke, on lipid lowering drug, taking drug associated with hypertriglyceridemia (corticosteroid, diuretics, Beta blocker), having other medical condition eg. chronic renal disease, hepatocellular disease, nephrotic syndrome, hypothyroidsm and patients attendant not consented were excluded.

\section{Operational definitions for the study}

Hypertriglyceridemia

The definition of hypertriglyceridemia is based on a classification in the Third report of the National Cholesterol Educational Program and the Adult Treatment Panel (NCEP-ATP III, USA) ${ }^{17}$.
Classification of serum triglyceride levels, according to the NCEP-ATP III: Normal - $150 \mathrm{mg} / \mathrm{dl}$; Border line high - 150 to 199 mg/dl; High- 200 to 499 mg/dl and Very high- $500 \mathrm{mg} / \mathrm{dl}$.

\section{Ischemic stroke}

Stroke is defined by the World Health Organization as a clinical syndrome consisting of 'rapidly developing clinical signs of focal (at times global) disturbance of cerebral function, lasting more than $24 \mathrm{~h}$ or leading to death with no apparent cause other than that of vascular origin ${ }^{18}$. Ischemic stroke or Cerebral infarction is mostly due to thromboembolic disease secondary to atherosclerosis in the major extracranial arteries (carotid artery and aortic arch $)^{1}$

Type 2 diabetes mellitus

Diabetes is a clinical syndrome characterised by hyperglycemia due to absolute or relative deficiency of insulin. In type 2 diabetes mellitus there is a combination of resistance to the action of insulin in liver and muscle together with impaired pancreatic beta cell function leading to relative insulin deficiency ${ }^{19}$.

\section{Detailed procedure}

Data regarding, sex and risk factors like BMI, life style, habit of smoking, betel nut chewing and alcohol intake, biochemical variables (fasting and post-prandial blood glucose and lipid profile), were recorded using predesigned semi-structured questionnaire. Details about the nature and purpose of the study were briefed to the patients and/ or their legitimate attendant and written consent was obtained from participant/ attendant. Basic principles of research ethics according to $52^{\text {nd }}$ WMA declaration of Helsinki'2000 \& CIOMS guidelines was maintained during the research processes.

\section{Outcome variables}

Age, sex, obesity, life style and, habit of smoking, betel nut and alcohol intake, doppler study of carotid vessels, triglyceride.

\section{Statistical analysis}

Data were expressed as mean $\pm S D$, range (minimum-maximum) and number (percent) as appropriate. Statistical analyses were performed using Statistical Program for Social Science (SPSS) 
for Windows, Version 16.0 (SPSS, Inc. Chicago III).Statistical tools Unpaired Student's-'t' test and Chi-squared test (Fisher Exact) were used to calculate statistical difference between groups as applicable. A p value $<0.05$ was taken as level of significance.

\section{Results:}

Male female distribution of the study subjects is shown in Figure 1. Of the 50 subjects in Group I male subjects constituted $56 \%$ and female $44 \%$ and in Group II the distribution is $53.3 \%$ and $46.7 \%$. This distribution does not show any significant association.

Mean $( \pm S D)$ age (yrs) is $61.0 \pm 10.6$ and $57.0 \pm 12.3$ in Group I and Group II respectively $(p=0.128)$. Distribution of subjects in percent in the two groups is shown in Figure II. Around sixty percent of the study subjects in the two groups are in age groups of 50-70 yrs. The distribution does not show significant association.

Clinico-biochemical data is shown in Table I. Age, $\mathrm{BMI}$ and blood pressure of the two groups does not show any statistical significance. Serum triglyceride and total cholesterol level are significantly high in the Group I compared to Group II ( $p=0.024$ and 0.009 respectively). LDL-c in the two groups do not show

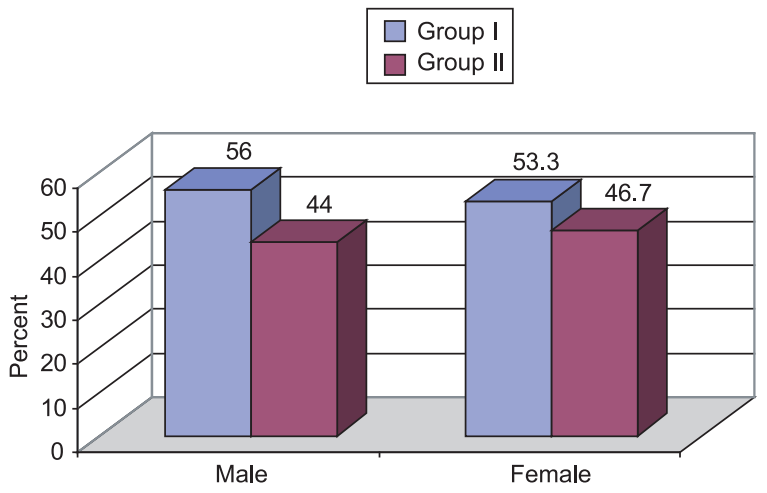

Fig.-1: Distribution (percent) of male and female subjects of the two groups (Group 1, acute ischemic stroke and Group II, without ischemic stroke). any significant difference $(p=0.828)$. HDL-c level is significantly lower in the Group I compared to the Group II ( $p=0.017)$. (Table I).

Distribution of the subjects with or without known risk factors in the two groups is shown in Table II. None of the subjects in the two groups has positive history of drinking alcohol. Body weight, physical activity level, smoking and betel nut chewing do not show any significant association with ischemic stroke. In Group I 88\% of the subjects with acute ischemic stroke has triglyceride level above the cut-off compared to $70 \%$ in the Group II which also do not show significant association $(p=0.079)$ (Table II).

Study variables are analyzed on the basis of serum triglyceride status (Table III). Sedentary life style and presence of carotid artery atherosclerotic changes are significantly associated with hypertriglyceridemia $(p=0.006$ and 0.004 respectively). Serum triglyceride level (mean $\pm S D$ ) is also looked into on the basis of presence or absence of risk variables. In the Group I subjects having carotid atherosclerotic changes and overweight-obesity (BMI $25 \mathrm{Kg} / \mathrm{m}^{2}$ ) has significantly high level of serum triglyceride level $(p=0.047$ and 0.035 ) (Figure IIIA and E respectively).

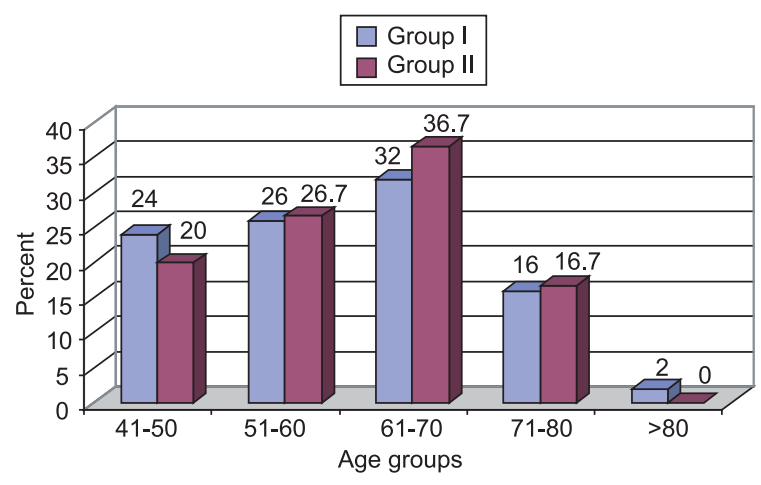

Figure 2: Distribution (percent) of subjects of the two groups (Group 1, acute ischemic stroke and Group II, without ischemic stroke) in different age groups. 

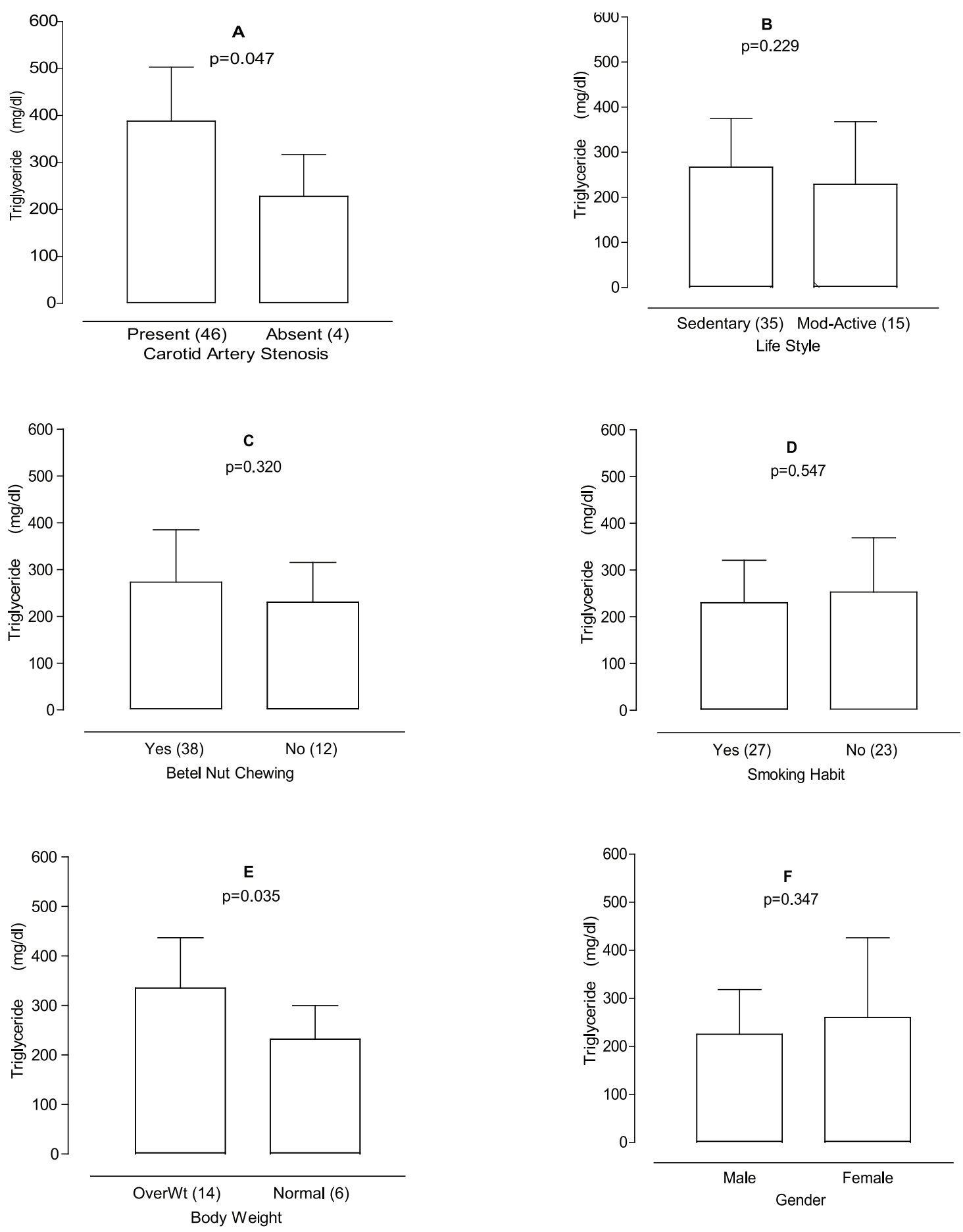

Fig.-3: Serum triglyceride $(\mathrm{mg} / \mathrm{dl})$ is shown in acute ischemic stroke patients with T2DM on the basis of carotid artery stenosis $(A)$, life style $(B)$, habit of betel nut chewing $(C)$ and smoking $(D)$, body weight $(E)$ and gender (F). 
Table-I

Clinico-biochemical variables of the study subjects

\begin{tabular}{lccc}
\hline Variables & Group I $(\mathrm{n}=50)$ & Group Il $(\mathrm{n}=30)$ & P value \\
\hline Age $(\mathrm{yrs})$ & $61.0 \pm 10.6$ & $57.0 \pm 12.3$ & 0.128 \\
BMl $\left(\mathrm{Kg} / \mathrm{m}^{2}\right)$ & $25.7 \pm 2.4$ & $24.8 \pm 3.2$ & 0.157 \\
Blood pressure $(\mathrm{mmHg})$ & & & \\
Systolic & $135 \pm 11$ & $140 \pm 13$ & 0.079 \\
Diastolic & $86 \pm 8$ & $83 \pm 10$ & 0.144 \\
Triglyceride $(\mathrm{mg} / \mathrm{dl})$ & $241 \pm 56$ & $217 \pm 102$ & 0.024 \\
T cholesterol $(\mathrm{mg} / \mathrm{dl})$ & $197 \pm 62$ & $165 \pm 26$ & 0.009 \\
LDL-c $(\mathrm{mg} / \mathrm{dl})$ & $101 \pm 45$ & $98 \pm 42$ & 0.828 \\
HDL-c $(\mathrm{mg} / \mathrm{dl})$ & $43.8 \pm 34.4$ & $60.0 \pm 15.0$ & 0.017 \\
\hline
\end{tabular}

Data is expressed as mean $\pm S D$. Unpaired Student's- 't' test is performed to calculate statistical difference between two groups. $\mathrm{P}<0.05$ is taken as level of significance.

Table-II

Distribution of the study subjects in the two groups on the basis of risk variables

\begin{tabular}{lccccc}
\hline Variables & \multicolumn{2}{c}{ Group I } & \multicolumn{2}{c}{ Group II } & p value \\
& $\mathrm{n}$ & $\%$ & $\mathrm{n}$ & $\%$ & \\
\hline Body weight & & & & & \\
Over weight-obese & 6 & 70.0 & 18 & 60.0 & 0.470 \\
Normal body weight & & 30.0 & 12 & 40.0 & \\
Smoking & 27 & 54.0 & 20 & 66.7 & 0.265 \\
Yes & 23 & 46.0 & 10 & 33.3 & \\
No & & & & & \\
Betel Nut chewing & 38 & 76.0 & 19 & 63.3 & 0.225 \\
Yes & 12 & 24.0 & 11 & 36.7 & \\
No & & & & & \\
Life style & 35 & 70.0 & 23 & 76.7 & 0.517 \\
Sedentary & 15 & 30.0 & 7 & 23.3 & \\
Moderately active & & & & & \\
Serum triglyceride & 44 & 88.0 & 21 & 70.0 & 0.079 \\
High (>150 mg/dl) & 6 & 12.0 & 9 & 30.0 & \\
Normal (<150 mg/dl) & & &
\end{tabular}

Data are expressed as number (percent).

Chi-squared test is performed to calculate statistical association for ischemic stroke. $\mathrm{P}<0.05$ is taken as level of significance. Over weight-obese, BMI $25 \mathrm{~kg} / \mathrm{m}^{2}$; Normal body weight, BMI $<25 \mathrm{Kg} / \mathrm{m}^{2}$ 
Table-III

Distribution of subjects with acute ischemic stroke (Group I) on the basis of different risk variables

\begin{tabular}{lccc}
\hline Variables & \multicolumn{2}{c}{ Serum triglyceride $(\mathrm{mg} / \mathrm{dl})$} & P value \\
& $>150 \mathrm{mg}$ & $<150 \mathrm{mg}$ & \\
& $\mathrm{N}(\%)$ & $\mathrm{N}(\%)$ & \\
\hline Gender & & & \\
Male $(n=28)$ & $24(87.7)$ & $4(14.3)$ & 0.683 \\
Female $(n=22)$ & $20(90.9)$ & $2(9.1)$ & \\
Body weight & $12(85.7)$ & $2(14.3)$ & 0.989 \\
Overweight-obese $(n=14)$ & $5(83.3)$ & $1(16.7)$ & \\
Normal $(n=6)$ & $24(88.9)$ & $3(11.1)$ & 0.898 \\
Smoking & $20(87)$ & $3(13)$ & \\
Yes $(n=27)$ & $35(92.1)$ & $3(7.9)$ & 0.141 \\
No $(n=23)$ & $9(75)$ & $3(25)$ & \\
Betel nut chewing & & & \\
Yes $(n=38)$ & $34(97.1)$ & $1(2.9)$ & 0.006 \\
No $(n=12)$ & $10(75)$ & $5(25)$ & \\
Physical activity & & & \\
Sedentary & $43(93.5)$ & $3(6.5)$ & 0.004 \\
Moderately active & $1(25)$ & $3(75)$ & \\
Carotid artery stenosis & & \\
Yes $(n=46)$ & & \\
No $(n=4)$ & &
\end{tabular}

Data are expressed as number (percent).

Chi-squared test is performed to calculate statistical association for ischemic stroke. $\mathrm{P}<0.05$ is taken as level of significance. Over weight-obese, BMI $25 \mathrm{~kg} / \mathrm{m}^{2}$; Normal body weight, $\mathrm{BMI}<25 \mathrm{Kg} / \mathrm{m}^{2}$

\section{Discussion}

This observational study was carried out with an aim to explore the association, if any, between triglyceride and ischemic stroke in diabetic patients. A total number of 50 type 2 diabetic patient with acute ischemic stroke admitted in the department of Neurology, BIRDEM General Hospital. Thirty (30) T2DM patients without ischemic stroke served as controls.

The present study demonstrated that about $60 \%$ of cases in both the croups are in 50-70 years age group. Although in an earlier study it is reported that $36.2 \%$ developed stroke in more than 60 years $^{20}$. Mean age $(61.0 \pm 10.6)$ of the patients with ischemic stroke which is consistent with previous reports ${ }^{11,21}$. However, some authors reported relatively lower mean age (yrs) at the time of the events ${ }^{22,23}$.

The incidence of ischemic stroke in the present study is $56.0 \%$ and $44.0 \%$ in male and female respectively. Male to female ratio is 1.3:1 in ischemic stroke. This observation is consistent with number of studies which showed similsr male to female ratio $^{8,19,24}$. Although, male preponderance was high (4.9:1) compared to their female counterpart in one report ${ }^{25}$.

In the present study $70.0 \%$ were overweight (BMI $25 \mathrm{~kg} / \mathrm{m}^{2}$ ) in Group I and 60.0\% in Group II. More than a half $(54.0 \%)$ of the patients was smoker in Group I and $66.7 \%$ in Group II. More than three fourth $(76.0 \%)$ of the patients had habit of betel nut chewing in Group I and relatively low (63.3\%) in Group II. Sedentary lifestyle was $70.0 \%$ observed in Group I and $76.7 \%$ in Group II. These features did not show any statistical association which indicated that the risk factors were almost consistent between two groups. Marked hypertriglyceridemia in type 2 diabetes mellitus and presence of other predisposing factors which include chronic insulin deficiency, age, obesity, sedentary lifestyle, 
medications for other comorbid conditions were reported earlier ${ }^{21}$. Diabetes mellitus, hypertension, dyslipidemia, smoking, family history of CAD and age more than 60 years were considered as conventional risk factors for ischemic stroke. History of smoking was present in $24 \%$ cases.

Hypertriglyceridemia (>150 mg/dl) was present in almost ninety percent $(88.0 \%)$ of the patients of Group I and $70.0 \%$ in Group II and significantly higher mean triglyceride level in Group I compared to the counterpart Group II $(p=0.024)$. However in one study relatively low mean triglyceride level $(145.0 \pm 85.3 \mathrm{mg} / \mathrm{dl})$ in the ischemic stroke group compared the controls $(241.0 \pm 103 \mathrm{mg} / \mathrm{dl})^{23}$. In the present study serum total cholesterol is also found to be significantly higher $(p=0.009)$ in the ischemic stroke group. However, LDL-c was almost similar in the two groups. But HDL-c was significantly lower $(p=0.017)$ in the ischemic stroke group (Table I). One particular study demonstrated significantly higher level of total cholesterol (mg/dl) $[217.7 \pm 49.7$ vs 202.3 $\pm 45.8 ; p=0.001]$ and lower HDL-c [ $41.5 \pm 12.1$ vs $45.9 \pm 17.7 ;(p=0.010)$ in stroke/TIA patients compared to the control ${ }^{21}$. The authors, however, also demonstrated significantly higher LDL-c in the ischemic stroke group. Although findings in the present study were consistent regarding lipid levels in the other reports ${ }^{9,11}$.

Proportion of male and female patients with hypertriglyceridemia (>150 mg/dl) was relatively high and almost similar frequency $[85.7 \%$ and $90.9 \%$ respectively]. The mean triglyceride was found $225.07 \pm 93.09 \mathrm{mg} / \mathrm{dl}$ in male and $260.32 \pm 166.43 \mathrm{mg} /$ $\mathrm{dl}$ in female patients, which was almost similar between male and female patients. Similar observations regarding the triglyceride (TG) level between male and female were also demonstrated in two different reports ${ }^{13,26}$.

Height and weight could be measured only 20 patients in the present study. Out of which 14 patients were overweight-obese (BMI $>25 \mathrm{~kg} / \mathrm{m}^{2}$ ) and 6 patients had normal body weight $(\mathrm{BMl}<25$ $\mathrm{kg} / \mathrm{m}^{2}$ ). The mean triglyceride level was found $335.0 \pm 101.5 \mathrm{mg} / \mathrm{dl}$ in over weighted patients and $231.6 \pm 67.9 \mathrm{mg} / \mathrm{dl}$ in normal body weight patients, which was significantly higher in over weight-obese patients ( $p=0.035$, Figure IIIE). This was supported in the study reported previously who have also observed marked hypertriglyceridemia associated with obesity ${ }^{5}$.

Habit of smoking and betel nut chewing in the present study did not show any statistical association with ischemic events and those having the risk factor present also had almost similar level of serum triglyceride level (Table III and Figure III). These features are supported by other authors.

The present study demonstrated that $97.1 \%$ of the patients with ischemic stroke had sedentary life and $66.7 \%$ of them had hypertriglyceridemia. Although serum triglyceride level between patients having sedentary lifestyle and moderately activity level did not show statistical difference. Although earlier it was suggested suggested that hypertriglyceridemia indicates the presence of predisposing factors like sedentary lifestyle and/or late expression of other genetic lipid disturbances ${ }^{5}$.

Of the total ischemic stroke subjects atherosclerotic changes in carotid vessels was observed in 46 subjects and of these $93.5 \%$ had hypertriglyceridemia. However, of the 4 subjects who had no atherosclerotic changes only 1 had hypertriglyceridemia and this distribution showed significant association $(p=0.004)$. Triglyceride level was also significantly high in those with presence of atherosclerotic changes compared to the counterpart $(p=0.047)$ (Figure IIIA). This observation possibly signifies that hypertriglyceridemia possibly accentuate progression of atheroscrerotic changes in carotid vessels and incur additional risk for ischemic stroke in the diabetic patients.

\section{Conclusions:}

Data concluded that hypertriglyceridemia is relatively common among the diabetic patients even in patients with apparently good glycemic control and possibly incur added risk for ischemic stroke in these patients. However, further studies are needed involving optimum number of patients to substantiate this finding and conclusively comment on the issue and to design effective prevention program to reduce the cerebrovascular morbidity and mortality. 


\section{References:}

1. Allen CMC, Lueck CJ, Dennis M. 2010. Neurological disease. In; Colledge NR, Walker $\mathrm{BR}$, Ralston SH, editors, Davidson's Principles and Practice of Medicine, $21^{\text {st }}$ ed. Edinburgh: Churchill Livingstone; 2010.p 1180-90.

2. Koren-Mora, N, Tanne D, Graff E, Goldbourt $U$, Low- and high-density lipoprotein cholesterol and ischemic cerebrovascular disease: the bezafibrate infarction prevention registry. Arch Intern Med 2002; 62: 993-9.

3. Sacco RL, Adams R, Albers G, Albert MJ, Benavente O, Furie $\mathrm{K}$ et al. Guidelines for Prevention of Stroke in Patients with Ischemic Stroke or Transient Ischemic Attack. Stroke 2006; 37: 577-617.

4. Coughlan BJ, Sorrentino MJ, Does hypertriglyceridemia increase risk for CAD? Postgrad Med 2000; 108: 77-84.

5. Kreisberg RA. Diabetic Dyslipidemia. Am J Cardiol 1998; 82: 67-73.

6. Anderson RA, Evans ML, Ellis GR, Graham $J$, Morris K, Jackson SK et al. The relationships between post-prandial lipaemia, endothelial function and oxidative stress in healthy individuals and patients with type 2 diabetes. Atherosclerosis 2001, 154: 475-83.

7. Mack WJ, Krauss RM, Hodis HN. Lipoprotein subclasses in the Monitored Atherosclerosis Regression Study (MARS). Treatment effects and relation to coronary angiographic progression. Arterioscler Thromb Vasc Biol. 1996;16: 697-704

8. Lewis TV, Dart AM, Chin-Dusting JPF. Endothelium-Dependent Relaxation by Acetylcholine Is Impaired in Hypertriglyceridemic Humans With Normal Levels of Plasma LDL Cholesterol. J Am Coll Cardiol. 1999; 33: 805-12.

9. Abe Y, El-Masri, B, Kimball K, Pownall H, Reilly $\mathrm{CF}$, Osmundsen $\mathrm{K}$ et al. Soluble Cell Adhesion Molecules in Hypertriglyceridemia and Potential Significance on Monocyte Adhesion. Biol. 1998; 18, pp.723-31.
10. Hackman A, Abe Y, Insull W, Pownall H, Smith $\mathrm{L}$, Dunn $\mathrm{K}$ et al. Levels of Soluble Cell Adhesion Molecules in Patients with Dyslipidemia. Circulation. 1996; 93: 1334-38.

11. Papagianni A, Kokolin, E, Kalovoulos M, Vainas A, Dimitriadis C, Memmos D et al. Carotid atherosclerosis is associated with inflammation, malnutrition and intercellular adhesion molecule-1 in patients on continuous ambulatory peritoneal dialysis. Nephrol Dial Transplant, 2004;19: 1258-63.

12. Teno $\mathrm{S}$, Uto $\mathrm{Y}$, Nagashima $\mathrm{H}$, Endoh $\mathrm{Y}$, Iwamoto $Y$, Omori $Y$ et al. Association of Postprandial Hypertriglyceridemia and Carotid Intima-Media Thickness in Patients with Type 2 Diabetes. Diabetes Care. 2000; 23: 1401-06.

13. Rosenson RS, Shott S, Lu L, Tangney CC, Hypertriglyceridemia and other factors associated with plasma viscosity. Am J Med 2001;110: 488-92

14. Seplowitz, AH, Chien S, Smith FR. Effects of lipoproteins on plasma viscosity. Atherosclerosis, 1981; 38: 89-95.

15. Lindenstrom E, Boysen G, Nyboe J. Influence of total cholesterol, high density lipoprotein cholesterol, and triglycerides on risk of cerebrovascular disease: the Copenhagen city heart study. BMJ 1994; 309: 11-15.

16. Freiberg JJ, Tybjaerg-Hansen A, Jensen JS, Nordestgaard BG, Nonfasting triglycerides and risk of ischemic stroke in the general population. JAMA 2008; 18: 2142-52.

17. Freiberg Third Report of the National Cholesterol Education Program (NCEP) Expert Panel on Detection, Evaluation, and Treatment of High Blood Cholesterol in Adults (Adult Treatment Panel III) final report. Circulation 2002;106: 3143

18. Hatano S. Experience from a multicentre stroke register: a preliminary report. Bulletin of the World Health Organization, 1976; 54, 541-53. 
19. Frier BM, Fisher M. Diabetes Mellitus. In; Colledge NR, Walker BR, Ralston SH, editors, Davidson's Principles and Practice of Medicine, $21^{\text {st }}$ ed. Edinburgh: Churchill Livingstone; 2010; 1180-90.

20. Alam B, Habib M, Qurashi FA, Haque B, Hoque A, Mohammad QD. Stroke Evaluation of risk factors. Bang J Neurosci. 1999; 15(1/2): 1418.

21. Laloux P, Galantl L, Jamart J. Lipids in ischemic stroke subtypes. Acta Neurol Belg 2004; 104: 13-19

22. Rahman KM, Haque A, Ullah AKMA, Khan RK, Alam MB. Study of modifiable risk factors for ischemic stroke. Ban J Neurosci 2001; 17: 6-9.

23. Kumar V, Madhu SV, Singh G, Gambhir JK. Post-Prandial Hypertriglyceridemia in Patients with Type 2 Diabetes Mellitus with and without Macrovascular Disease. JAPI 2010;58:603-07

24. Venkatesh G, Ramanathan M, Reddy CJ, Vijaya RKN. Prevalence of Coronary Risk Factors in Indian Population vis-á-vis Framingham Cohort. Indian Heart J 2005; 57 : 543-554.

25. Chowdhury SGM. Stroke in patients having inadequate or irregular antihypertensive therapy. BMRC Bull 1990; 16: 52-7.

26. Antonios N, Angiolillo DJ, Silliman S.. Hypertriglyceridemia and Ischemic Stroke. Eur Neurol 2008; 60: 269-78.

27. Mukhopadhyay P, Satpati AK, Khan AK, Chaterjee BK, Sadhukhan S. Tobacco Chewing - A Potential Cardiovascular Risk Factor: A Comparative Study in a Tertiary Center. Indian Heart J 2005; 57: 543-54. 